\title{
Zum Informationswert der deutschen und tschechischen Satzspitzen
}

\section{Hana Peloušková}

This article presents the second part of a contrastive study on word order and functional sentence perspective, which is based on authentic language material from the Czech-German parallel corpus InterCorp.

The introductory research forming part of a more complex project deals with elements that occupy the first sentence position in German and in Czech, examines their frequency and looks for parallels and discrepancies in these two languages.

The subsequent research deals with the first sentence positions in terms of functional sentence perspective. It describes and compares grammatical ("fixed"), thematic and rhematic sentence beginnings in both languages.

The results of the contrastive research will form a theoretical basis for recommendations for the treatment of word order and functional sentence perspective at different levels of foreign language teaching.

contrastive syntax - dependency grammar - word order - functional sentence perspective theme - rheme - corpus analysis - parallel corpus

Der Artikel stellt den zweiten Teil einer kontrastiven Teilstudie zum Thema Satzgliedstellung und funktionale Satzperspektive dar. Die Untersuchungen stützen sich auf authentische Belege aus dem tschechisch-deutschen Parallelkorpus InterCorp.

Im ersten, einleitenden Teil der Teilstudie wurden Elemente untersucht, die die deutschen und tschechischen Satzspitzen besetzen. Es wurde ihre Frequenz festgestellt und die sich daraus ergebenen Werte wurden für beide Sprachen verglichen.

Die daran anschließende Untersuchung konzentriert sich auf den Informationswert der an der Satzspitze stehenden Satzelemente in den beiden Sprachen. Es werden deutsche und tschechische grammatische („fixe“), thematische und rhematische Satzspitzen beschrieben und verglichen.

Die Ergebnisse der geplanten kontrastiven Untersuchungen sollen eine theoretische Basis für sprachdidaktische Empfehlungen für die Behandlung der Satzgliedstellung und der funktionalen Satzperspektive auf verschiedenen Niveaus des Fremdsprachenunterrichts schaffen.

kontrastive Syntax - Dependenzgrammatik - Wortfolge - funktionale Satzperspektive Thema - Rhema - Korpusanalyse - Parallelkorpus

\section{Zielsetzung und Eingliederung in den Forschungskontext}

Dieser Artikel stellt den zweiten Teil einer kontrastiven Teilstudie dar, die sich mit der Besetzung der Satzspitze im Deutschen und Tschechischen befasst. Diese Teilstudie ist Teil einer geplanten komplexeren Studie zur Problematik der Satzgliedstellung und der funktionalen Satzperspektive, die sich im Augenblick noch in Arbeit befindet. 
Im ersten, einleitenden Teil dieser Teilstudie wurden bereits das Vorkommen und die Frequenz der an den Satzspitzen stehenden Elemente im Deutschen und Tschechischen untersucht, und die sich daraus ergebenen Werte wurden für beide Sprachen verglichen. Es wurden markante Unterschiede festgestellt, wie z. B. beim Auftreten von pronominalen Subjekten oder finiten Verbformen. (vgl. Peloušková 2016: 101-117)

Einen einfachen Vergleich der am häufigsten vorkommenden Satzanfänge ermöglicht die folgende Tabelle:

\begin{tabular}{|l|l|l|}
\hline Konstruktion & Deutsch & Tschechisch \\
\hline Subjekt & $56 \%$ & $30 \%$ \\
\hline Angabe & $22 \%$ & $25 \%$ \\
\hline die übrigen Ergänzungen & $10 \%$ & $11 \%$ \\
\hline Verb & $3 \%$ & $24 \%$ \\
\hline
\end{tabular}

Tab. 1: Besetzung der deutschen und tschechischen Satzspitzen im Vergleich (Peloušková 2016: 106)

Im hier vorliegenden Teil der Gesamtstudie konzentrieren wir uns auf den Informationswert der deutschen und tschechischen Satzspitze.

Anschließend sollen sprachdidaktische Empfehlungen für die Behandlung dieser Problematik im Fremdsprachenunterricht formuliert sowie ergänzende Unterrichtsmaterialien erstellt werden.

Theoretischer Ausgangspunkt für diese Teilstudie ist die Valenztheorie. Die Untersuchungen stützen sich auf authentische Daten aus dem tschechisch-deutschen Parallelkorpus InterCorp. ${ }^{1}$

\section{2. "Fixe" und "aktuelle" Satzspitze}

Die Spitzenstellung von bestimmten Satzelementen kann genauso wie die Satzgliedstellung an anderen Satzpositionen (in der Terminologie von Štícha 2015) „fix“ (d. h. formal, grammatisch bedingt, stellungsfest) oder (nach einer anderen Terminologie $)^{2}$ „aktuell“(d. h. von der funktionalen Satzperspektive bestimmt) sein.

\section{1 "Fixe" Satzspitzen im Deutschen und im Tschechischen}

In den beiden Sprachen gibt es einige Konstruktionen, in denen die Satzspitze stabil und somit rein grammatisch bestimmt ist. Der Anteil von Belegen mit fixer

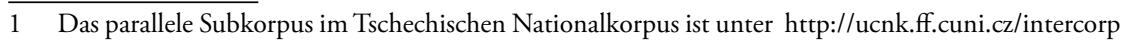
zugänglich.

2 Diese Begriffe verwenden u. a. Mathesius (1947), Eroms (1982), Štícha (2013). 
Satzspitze am untersuchten Material ${ }^{1}$ beträgt sowohl im deutschen als auch im tschechischen Teil des Korpus ca. 3 \%. Im Einzelnen handelt es sich dabei um drei verschiedene Konstruktionstypen.

\subsection{1 (Finite) Verben in Entscheidungsfragen und Imperativsätzen}

Sind Sie wirklich ein Geschäftsmann, Kleiner?

Jste byznysmen, mládenče?

Umarm die Mädchen von mir.

Obejmi za mè dèvčata.

Hier ist es aber notwendig, auf einen Unterschied zwischen dem Deutschen und dem Tschechischen aufmerksam zu machen. Während die Satzspitze in deutschen Entscheidungsfragen und Imperativsätzen immer nur durch eine finite Verbform besetzt ist, stehen in tschechischen Entscheidungsfragen oft Partizipien am Satzanfang. Dazu kommt es in Sätzen mit manchen zusammengesetzten Verbformen, in denen die finite Form des Hilfsverbs klitischen Charakter hat und erst nach dem betonten Partizip folgt, $\mathrm{z}$. B.:

Möchtest du einen industriellen Gimpel auf irgendein Schmarrnpatent fangen ... Chtěl bys chytit prưmyslovou kavku na nějaký usmolený patent ... (Konditional)

\subsubsection{Fragewörter in Ergänzungsfragen}

Wie soll er denn schreiben, wenn du immer auf ihm herumreitest?

Jak může psát, když na něj pořád dorážíšs?

Die Satzspitze ist hier im Deutschen und Tschechischen identisch.

\subsubsection{Weitere Typen von fixer Satzspitze im Deutschen}

Spezifisch für das Deutsche (im Vergleich zum Tschechischen) ist die Spitzenstellung der finiten Verbformen u. a. in uneingeleiteten Wunschsätzen und manchen Ausrufesätzen. ${ }^{2}$

Hätte ich es nur gewusst!

Kdybych to (byla) vèděla! / Kéž bych to (byla) vèdèla!

Ist das heute wieder eine Hitze!

To je dnes ale horko!

11000 per Zufallsstichprobe ausgewählte Parallelbelege aus dem Parallelkorpus InterCorp.

2 Die angeführten Beispiele sind konstruiert. In der untersuchten Stichprobe war dieser Konstruktionstyp nicht vertreten. 
In den tschechischen Entsprechungen kommen an der Satzspitze andere Elemente vor als in den deutschen Konstruktionen (Einleitungswörter, Partikeln). In der analysierten Stichprobe gab es keine Belege für diese Art von Satzspitze.

Im Einklang mit der statistischen Beschreibung der Besetzung der Satzspitze erwähnen wir hier die fixen Einleitungen und Verb-erst-Stellungen in uneingeleiteten Nebensätzen nicht, weil wir vorangestellte Nebensätze als an die Satzspitze gestellte Konstituenten/Satzglieder auffassen.

In der gesprochenen Sprache und auch in der spontan geschriebenen Sprache (wie beim Skypen oder Chatten) wird das pronominale Subjekt (ich, $d u$, wir...) an der Satzspitze häufig nicht realisiert. In diesem Fall steht dann das finite Verb an der Satzspitze:

bab jetzt was umgestellt - funktionierts?

bin ja am Computer eine volle Niete

macht doch nichts

Wünsche euch noch einen schönen Tag!"

\subsection{Funktionale Satzperspektive}

Die Erscheinung, die in der Fachliteratur mit den Termini „funktionale Satzperspektive“, „Mitteilungsperspektive“, „aktuelle Satzgliederung“, „kommunikative Gliederung“, „Thema-Rhema-Gliederung“, „topic-comment structure”, „aktuální členění věty“ und anderen bezeichnet wird, charakterisiert die Duden-Grammatik (2005) wie folgt:

Das Model der Funktionalen Satzperspektive (FSP) (auch als Thema-Rhema-Gliederung (TRG) bezeichnet) untersucht, wie kontextuelle Kohäsionsmittel und satzsyntaktische Zeichen wie Intonation und Wortstellung bei der Verteilung von Informationen in Sätzen und Texten zusammenwirken. (Duden 2009: 1119)

Ähnlich definieren diese Erscheinung auch andere Quellen: Štícha (2011, 2013, 2015), Zifonun (1997), Eroms (1986), Lötscher (1983), Uhliŕová (1983), Firbas (1982), Beneš (1967) u. a.

An der funktionalen Satzperspektive (TRG) beteiligen sich mehrere Faktoren. ${ }^{2}$ Diese Faktoren sind praktisch in allen Sprachen wirksam. Das, was die einzelnen Sprachen voneinander unterscheidet, ist der Anteil der einzelnen Faktoren an der Organisation von Aussagen.

1 Beispiele aus einer spontanen Skypekonversation

2 Dazu Mathesius (1947), Beneš (1969), Firbas (1982), Lötscher (1983), Svoboda (1989), Götze (1989) u. a. 


\subsubsection{Die Begriffe "Thema“ und "Rhema"}

Es gibt zahlreiche terminologische Entsprechungen dieser Begriffe, z. B.:

- psychologisches Subjekt - psychologisches Prädikat (Paul 1880/1975; Ammann 1928; vgl. auch Eroms 1986: 2-7),

- východisko/východiště (výpovědi) - jádro (výpovědi) (Mathesius 1947),

- theme - rheme, topic - comment (Daneš 1964),

- téma - réma (Uhlí̌ová 1987).

Definitionen für die oben genannten dichotomischen Begriffspaare wie z. B.:

- alt/nicht neu - neu,

- bekannt - unbekannt,

- given - not given,

- worüber gesprochen wird - was davon gesagt wird,

- Ausgangspunkt der Aussage - die eigentliche Aussage,

- Präsupposition - Assertion u. a.

werden oft als vereinfachend oder ungenau kritisiert. Eine Analyse bietet z. B. Eroms (1986: 1-29). Eroms definiert im Einklang mit Lötscher (1983) das Thema als „das der Mitteilung Zugrundeliegende/Besprochene“ und das Rhema als „das dazu Ausgesagte“ (Eroms 1986: 25).

\subsubsection{Die Position von Thema und Rhema}

In der Regel steht das Thema (Th) links und das Rhema (Rh) rechts im Satz (nach Mathesius die „objektive“ Abfolge - emotional neutral, merkmallos; demgegenüber bezeichnet er die Abfolge Rh - Th als „subjektiv“ - emotional gefärbt, merkmalhaft), aber es wirken natürlich auch andere Faktoren für die Abfolge von Elementen zusammen, wie der Konstruktionstyp und natürlich auch die Intonation bzw. der Satzakzent.

In der merkmallosen Abfolge Th - Rh liegt das Mitteilungs- und Intonationszentrum am Satzende (automatisierte Intonation), in der merkmalhaften Abfolge Rh - Th am Satzanfang.

Die Th - Rh Abfolge wird im Allgemeinen bei objektiver, sachlicher Mitteilung, die Rh - Th Abfolge bei subjektiver, expressiver Ausdrucksweise verwendet.

In der gesprochenen Sprache sind beide Abfolgetypen deutlich intonatorisch voneinander geschieden, in der geschriebenen Sprache ist das Mitteilungszentrum oft textimmanent oder graphisch vorbestimmt, z. B.:

Viš, co tu mám? Rum pro tebe.

Weißt du, was ich hier habe? Rum für dich. 


\subsubsection{Besetzung der Satzspitze aus der Sicht der FSP}

Aus der Sicht der FSP kann die Satzspitze sowohl mit einem Thema als auch mit einem Rhema besetzt werden.

\subsubsection{Thematische Satzspitze}

Bei der weiteren Klassifizierung von Sätzen mit thematischer Satzspitze spielt die Kontextgebundenheit der Sätze eine wesentliche Rolle.

Je nach der Eingliederung des Satzes in einen Kontext in sind folgende Haupttypen zu unterscheiden.

\subsection{Kontextfreie Sätze}

- Abgeschlossene Informationen (de facto Antworten auf die Frage „Was gibt es Neues?")

Heute haben wir eine schwierige Aufgabe zu Ende gebracht.

Dnes jsme dokončili obtižný úkol.

Meine Frau ist gestorben, der Sohn hat die Kanzlei übernommen, ich bin alt geworden. Manželka zemrela, syn po mně prevzal kancelár a já jsem zestárl.

- Definitionen, Lehrsätze, gesetzliche Bestimmungen, Sentenzen, Sprichwörter:

Alle Bürger sind vor dem Gesetz gleich.

Vsichni občané jsou si préd zákonem rovni.

Aller Anfang ist schwer.

Každý začátek je těžký.

In kontextfreien Sätzen ist die Funktion des Themas im Vorfeld sehr deutlich ausgeprägt (d. h. was besprochen wird). Das Thema provoziert zur Frage „Was ist damit?", die dann mit dem Rhema beantwortet wird. Tschechische kontextfreie Sätze haben analoge Satzspitzen.

\subsection{Kontextgebundene Sätze ${ }^{1}$}

Die Satzspitze in kontextgebundenen Sätzen teilt Beneš (1971: 168-170) in drei Kategorien auf.

1 Vgl Beneš (1971: 167 ff). Kontextgebundene Sätze könnten wir in Anlehnung an Beneš weiter in rechtseitig, linksseitig und beiderseitig kontextgebundene Sätze unterteilen. 
- Thematische Komponenten von geringem Mitteilungswert, die auf verschiedene Textelemente zurückweisen, durch Pronomen, Pronominaladverb, durch synonyme Substitution oder Wiederholung ausgedrückt, z. B.:

Dr. Griffin sagte, ich soll einen Spezialisten aufsuchen. Er machte den Termin. Doktor Griffin ř́kal, že bych mël jit ke specialistovi. Domluvil mi vyšetřeni. ${ }^{1}$ ... bei dem sie und Onkel Frederick aus angemessener Entfernung in einer Kutsche über uns wachten. Danach aßen wir zu viert Eis unter Sonnenschirmen ...

... prìčemž mě se strýčkem Frederikem z určité vzdálenosti obezřetně sledovali ze svého kočáru. Poté jsme si všichni čtyři dali pod slunečniky zmrzlinu ...

- Thematische Komponenten von etwas höherem Mitteilungswert, die die Mitteilung ein wenig vorantreiben; die übrigen thematischen Komponenten von geringerem Mitteilungswert erscheinen erst nach dem Finitum. Diese Satzspitze kommt oft in Sachprosa vor, wo inhaltliche Zusammenhänge plastischer konturiert werden, aber auch in künstlerischer Prosa und in der Poesie (aus rhythmischen Gründen), z. B.:

Für mich ist die Liebe zu Frauen immer ein reinigendes Anbeten gewesen, eine steile Flamme meiner Trübe entlodert, Beterhände zu blauen Himmeln emporgestreckt.

Pro mě láska k ženám vždycky byla očistné zbožňování, strmý plamen vyšlehnuvší $\mathrm{z}$ mé zádumčivosti, $\mathrm{k}$ modlitbě sepnuté ruce vztáhnuté $\mathrm{k}$ blankytným nebesům.

- „Füllwörter“

Mit diesem Begriff bezeichnet Beneš den Platzhalter es (auch Auftakt-es), wie etwa in Es war einmal ein König.

Es als Platzhalter hat aber eine andere als satzverknüpfende Funktion; es ist wirklich nur ein „Füllwort“ ohne Mitteilungswert. $\mathrm{Zu}$ solchen Elementen kommen wir später.

\subsubsection{Rhematische Satzspitze}

Theoretisch können alle vorfeldfähigen Elemente als Rhema an der Satzspitze erscheinen. Bei manchen Elementen, z. B. bei pronominalen Subjekten oder Angaben, kann aber in der Regel nur die Betonung die rhematische Funktion verdeutlichen, deshalb werden in der geschriebenen Sprache meistens andere Mittel zur Rhematisierung bevorzugt.

Die Häufigkeit der rhematischen Satzspitze ist in verschiedenen Textsorten unterschiedlich. Selten ist die rhematische Spitzenstellung in Sachtexten, in Fachsprachen oder auch in der Sachprosa. Die Spitzenstellung des Rhemas dient

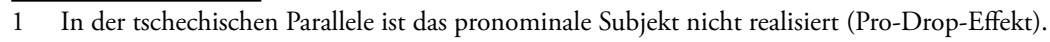


hier meistens ohne jede emotionale Färbung als Mittel der besonders starken Hervorhebung. Für expressive Ausdrucksweise ist dagegen die rhematische Spitzenstellung charakteristisch und erscheint daher sehr häufig in der künstlerischen Prosa, in der Poesie, in Texten der Boulevardpresse und natürlich in der Alltagssprache und in der Nachahmung der gesprochenen Sprache in der Belletristik.

Als rhematisch (ohne eine absolute Sicherheit) wurden im analysierten Belegmaterial folgende Elemente identifiziert:

\subsubsection{Subjekt}

- Pronominales Subjekt

Niemand erschien zu der Totenwache.

Nikdo neprǐsel posedèt u zesnulého.

Jeder kann sich beschneiden lassen, zum Beispiel aus hygienischen Gründen.

Obřezat se prece mỉze z hygienických divodù nechat každý.

In der tschechischen Parallele zum zweiten Beispiel nimmt das Subjekt die übliche rhematische Position ein.

Ich bin es, der von euch lernen muß!

Já se prece musim učit od vás!

Die an die Satzspitze gestellten Personalpronomina als Subjekte sind im Deutschen in den meisten Fällen thematisch. Hier dient die Konstruktion Ich bin es zur Hervorhebung des Rhemas.

Dagegen sind Personalpronomina als Subjekte im Tschechischen viel seltener ${ }^{1}$ und in der geschriebenen Sprache eher merkmalhaft. In der gesprochenen Sprache sind sie aber üblich:

Ich bin glücklich.

Já jsem štastná.

In diesen Parallelen lässt sich das Subjekt als rhematisch interpretieren, obwohl natürlich der Kontext und die Intonation eine entscheidende Rolle bei der Bestimmung des Informationswertes spielen.

- Substantivisches Subjekt

Ein Polizist stand übrigens an der Straßenecke und blickte zur Seite. Na rohu ulice stál policajt a koukal samozrejmè jinam.

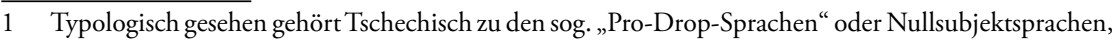
in denen Personalpronomina in der Subjektposition nicht realisiert werden müssen. Die Besetzung der Subjekt-Position durch ein Personalpronomen ist daher meistens nicht neutral. 
Ein Kommando deutscher Offiziere ist irgendwann nachts mit Fallschirmen in der Nähe von Moskau abgesetzt worden.

Někdy v noci bylo v blizkosti Moskvy vysazeno komando nèmeckých di̊stojnikì.

Ein Schrei war zu hören.

Zaznèl výkřik.

Den rhematischen Charakter signalisiert in den deutschen Belegen auch der unbestimmte Artikel. In den tschechischen Parallelen steht das rhematische Subjekt meistens an der üblichen rhematischen Position (am Ende).

Kiefer war es, der es aussprach:

Byl to Kiefer, který to vyslovil:

Zur Rhematisierung des Subjektes dient hier wieder die Konstruktion $X$ war es. In der tschechischen Parallele besetzt das Subjekt wieder die rhematische Position am Ende.

2.2.2.2.2 Andere Kasusergänzungen

„Hübsches Boot", sagte Clay, während er an Bord ging.

„Pèkný člun“", prohodil Clay, jakmile vstoupil na palubu.

\subsection{Angaben}

Obne dich hätten wir es wirklich nicht geschafft.

Bez tebe bychom to nedokázali.

\subsection{Satzklammerelemente}

Relativ selten erscheinen zum Zweck einer Rhematisierung des Vollverbs auch Satzklammerelemente, Partizipien oder Infinitive, z. B.:

Gezeigt wird von Natonek, wie ...

Natonek ukazuje, jak ...

Die Spitzenstellung des Verbs ist in diesem tschechischen Satz nicht möglich.

\subsection{Elemente, die im Mittelfeld stellungsfest sind}

Generell gesagt erscheinen an der Satzspitze relativ oft rhematische Ergänzungen, die sonst im Mittelfeld stellungsfeste / „fixe“ Positionen einnehmen (müssen) und außer der Intonation über keine anderen Mittel für die Hervorhebung verfügen. 
Sehr anschaulich zeigt uns diese Satzelemente die folgende vereinfachte Fassung der graphischen Darstellung der Satzgliedstellung im Mittelfeld nach Griesbach (1986):

\begin{tabular}{|l|l|l|l|l|l|l|l|l|l|l|}
\hline 1 & 2 & 3 & 4 & 5 & 6 & 7 & 8 & 9 & 10 & 11 \\
\hline $\mathrm{n}$ & $\mathrm{a}$ & $\mathrm{d}$ & $\mathrm{N}$ & Angaben & $\mathrm{D}$ & $\mathrm{A}$ & & & & $\mathrm{P}, \mathrm{G}, \mathrm{Ri}, \mathrm{Si}$, Art, Ein, nom. Teile von FGV \\
\hline \multicolumn{1}{|l|}{ stellungsfest } & \multicolumn{1}{|c|}{ stellungsvariabel } & $\begin{array}{l}\text { offene } \\
\text { Stellplätze }\end{array}$ & stellungsfest \\
\hline
\end{tabular}

Tab. 2: Graphische Darstellung der Satzgliedstellung im Mittelfeld ${ }^{1}$

Der Tabelle ist zu entnehmen, dass es im Mittelfeld zwei stellungsfeste Bereiche gibt, und zwar am Anfang, nach dem ersten Klammerteil (in Sätzen ohne Satzklammer nach der finiten Verbform), und am Ende des Mittelfeldes, unmittelbar vor dem zweiten Klammerteil (in Sätzen ohne Satzklammer dann am Ende des Satzes). Den ersten stellungsfesten/fixen Bereich nehmen pronominale Elemente wie Personalund Reflexivpronomina ein, die an diesen Positionen klitischen Charakter haben. Ihre Reihenfolge ist festgelegt: $\mathrm{n}-\mathrm{a}-\mathrm{d} .^{2}$ Stark betont können sie (mit Ausnahme echter Reflexivpronomina, die nicht erststellungsfähig sind) als Rhema die Satzspitze besetzen, z. B.:

Mir is das von ihr gleich eingefalln, wie ich von der Feldpost erzählen gehört hab, obzwar es gar nichts mit der Feldpost zu tun hat.

Mně to vo ni napadlo hned, jak jsem slyšel mluvit vo polnich poštách, ačkoliv to nemá docela nic společnýho s feldpostama.

Die stellungsfeste/fixe Position vor dem zweiten Klammerteil besetzen dann im Mittelfeld die folgenden Elemente: Präpositionalergänzung, Genitivergänzung, Richtungsergänzung, Situativergänzung, Artergänzung, Einordnungsergänzung oder nominale Teile von Funktionsverbgefügen. Die Kookkurrenz dieser Elemente ist ausgeschlossen, z. B.:

- Artergänzungen

Anspruchsvoll waren sie allerdings nicht,

Nároky nemèli ovšem žádné,

- Genitivergänzungen

Des Mordes an Zottmann wird er verdächtigt?

Je podezrelý z vraždy na Zottmannovi?

1 Übernommen aus Peloušková (2009d: 48), vereinfacht und ergänzt nach Griesbach (1986).

2 Nominativ - Akkusativ - Dativ. Die Abkürzung für den Kasus ist jeweils klein geschrieben, denn es handelt sich um „kleine“, d. h. kurze, unbetonte (klitische) Wortformen. 
- Präpositionalergänzungen

Auf dich haben wir hier gerade gewartet!

Na tebe jsme tu zrovna čekali.

- Fixe Elemente

Obwohl Interrogativpronomina und -adverbien in Ergänzungsfragen und Imperativformen in Aufforderungssätzen im Deutschen und im Tschechischen „fixe“/grammatische Positionen an der Satzspitze einnehmen, sind sie vom Informationswert her rhematisch, ${ }^{1}$ z. B.:

Wie soll er denn schreiben, wenn du immer auf ihm herumreitest?

$J a k$ může psát, když na něj pořád dorážíšs?

Wer seid ihr, und wer ist euer Herr?

Kdo jste a kdo je vás pán?

Rutsch mal richtig auf den Knien herum und fleh ihn an, deinen alten Rauschebart.

Lez tady po kolenou a vzývej ho toho tvýho starýho fousáče!

\subsubsection{Weder thematische noch rhematische Satzspitze}

An der Satzspitze können auch Elemente ohne Mitteilungswert erscheinen. Die eigentliche thematische Basis im Satz (ausnahmsweise das Rhema) bildet dann das danach folgende Verb. Diese Elemente sind die in 2.2.2.3.1 bis 2.2.2.3.3 genannten.

\subsection{Platzhalter es (das expletive es, das Vorfeld-es, "dummy").}

Das Platzhalter-es ist ein nicht referenzieller Ausdruck, der an der Satzspitze steht und die formale Aufgabe hat, die erste, unbesetzte Position im Satz zu füllen, um die Verb-Zweitstellung im Aussagesatz zu garantieren. Dieses es erscheint:

- in aktivischen und passivischen Sätzen mit dem rhematisierten (verschobenen) Subjekt und in subjektlosen passivischen Sätzen, z. B.:

Es wird jetzt gleich der Mond aufgehen.

Každou chvilku už musi vyjit mèsic.

Es sollen in diesem Bericht Obszönitäten möglichst vermieden werden...

Tato zpráva by se mèla pokud možno vyhnout obscenitám...

... es wird intensiv mit ihnen gearbeitet.

... hojně se použivají.

1 Auf diese Tatsache macht auch Hoberg (1981: 173) aufmerksam. 
In der Alltagssprache erscheinen an der Satzspitze außer dem Platzhalter-es oft auch andere Elemente, die neben der grammatischen auch eine satzverknüpfende Funktion haben (v. a. da und nun), z. B.:

Da bleiben weder Hände noch Leinwand sauber.

To pak nezistanou cisté ani ruce, ani plátno.

Nun blieben sie stehen.

Zưstali stát.

- in Sätzen mit persönlichem Akkusativ oder Dativ, z. B.:

Es graute mir schon immer vor dir.

V̌̈dycky jsem se tè dèsila.

Bei der Permutation ist die Anwesenheit von es hinter dem finiten Verb immer fakultativ.

Mir graute (es) schon immer vor dir.

Im Tschechischen kommt kein Element mit dieser Funktion vor, denn im Tschechischen gibt es keine formale Forderung hinsichtlich der Verb-Stellung. Die tschechischen Parallelen beginnen am häufigsten mit Verben oder thematischen Kulissen, ${ }^{1}$ falls sie im Satz vorhanden sind.

\subsection{Formales Subjekt}

Das formale Subjekt ist ein semantisch leerer, nicht referenzieller, nicht weglassbarer und nicht ersetzbarer Bestandteil von Konstruktionen mit manchen Verben, z. B.:

Es goss, und es war nebelig.

Lilo a byla mlha.

Es gibt andere, überzeugendere politische Instrumente.

Existují jiné, přesvědčivější politické nástroje.

An der Satzspitze übernimmt das formale Subjekt die Rolle des Platzhalters. Falls die Satzspitze ein anderes Element besetzt, steht das formale Subjekt hinter dem finiten Verb oder hinter dem Einleitungswort. Formale Subjekte im Deutschen haben im Tschechischen keine Entsprechungen. Die äquivalenten Konstruktionen sind oft identisch aufgebaut, jedoch fehlt das formale Subjekt.

1 Der Begriff „thematische Kulisse“ stammt von Sgall (1980). 


\subsection{Korrelat es}

Das Korrelat es korreliert mit Subjektsätzen oder Infinitivkonstruktionen als Konkurrenzformen von Subjektsätzen und übernimmt an der Satzspitze die Rolle des Platzhalters, z. B.:

Es ist schwierig, ihnen durch das Angebot einer anderen Politik Einhalt zu gebieten. Neni snadné jim vzit vitr z plachet a nabidnout alternativni koncepci v jakékoli oblasti.

Auch im Tschechischen gibt es ein Korrelat (to), dieses kommt allerdings nur sehr selten an der Satzspitze vor:

To (vỉbec) neni snadné jim ...

\subsection{Zur Häufigkeit und Funktionen der Satzspitzen}

Es ist immer wieder versucht worden, bestimmte Zusammenhänge oder Relationen zwischen der Häufigkeit und den Funktionen oder dem Informationswert herzustellen.

Engel (1977), Beneš (1971), Hoberg (1981) u. a. warnen aber vor jeder Absolutierung oder Dogmatisierung der Postulate. Als ganz akzeptabel haben sich die folgenden Behauptungen erwiesen:

(1) Je häufiger im Vorfeld, desto thematischer.

Es wird also ein Zusammenhang zwischen hoher Frequenz und thematischer Funktion hergestellt (z. B. Hoberg 1981: 174). Wie bereits erwähnt, kommen in solchen Kontexten im Deutschen substantivische und pronominale Subjekte sowie gewisse Angaben vor, im Tschechischen substantivische Subjekte, manche Angaben und Verben.

(2) Der Hervorhebungsgrad von Elementen scheint mit der Wahrscheinlichkeit des Auftretens im Vorfeld zusammenzuhängen (vgl. Engel 1977: 502), d. h. je seltener, desto rhematischer.

(3) Man nimmt oft an, dass ein bestimmtes Satzglied umso mehr im Vorfeld stilistisch auffällt, je seltener es dort auftritt (vgl. Beneš 1971: 165).

(4) Nur teilweise und grob gesehen (vor allem für Subjekte) gilt auch: Je häufiger im Vorfeld, desto mehr satzverknüpfend (vgl. Hoberg 1981: 175; vgl. auch Svoboda 1989: 9-24).

1 Konstruiertes Beispiel. 


\section{Schlussfolgerung}

Die Analyse hat bestätigt, dass die "fixen“ (grammatischen) Satzspitzen im untersuchten Material in den beiden Sprachen nur marginal vertreten sind, jedoch weisen sie bis auf Ausnahmen (z. B. uneingeleitete Wunschsätze) beträchtliche Übereinstimmungen auf.

Auch die Überlegungen über den Informationswert der Satzspitzen haben viele Gemeinsamkeiten der beiden Sprachen zutage gefördert. Daneben wurde aber auch auf unterschiedliche Präferenzen bei der Spitzenstellung aufmerksam gemacht und es wurden Zusammenhänge zwischen der Häufigkeit und dem Informationswert und damit zusammenhängende Diskrepanzen zwischen beiden Sprachen festgestellt. Auf diese Problematik muss noch tiefgehender eingegangen werden.

Die Formulierung von sprachdidaktischen Empfehlungen für die Behandlung dieser Problematik im Fremdsprachenunterricht kann erst nach dieser eingehenderen Analyse erfolgen.

\section{Literatur}

Beneš, Eduard (1971): Die Besetzung der ersten Position im deutschen Aussagesatz. In: Fragen der strukturellen Syntax und der kontrastiven Grammatik. Sprache der Gegenwart 17. S. $160-182$.

Beneš, Eduard (1967): Die funktionale Satzperspektive im Deutschen. In: Deutsch als Fremdsprache 4/1/1967. S. 23-28.

Beneš, Eduard (1964): Die Verbstellung im Deutschen von der Mitteilungsperspektive her betrachtet. In: Muttersprache 74/1/1964. S. 9-21.

Beneš Eduard (1959): Začátek německé věty $\mathrm{z}$ hlediska aktuálního členění větného. In: Časopis pro moderní filologii 41/1959. S. 205-217.

Daneš, František (1968): Typy tematických posloupností v textu (na materiále českého textu odborného). In: Slovo a slovesnost 29/2/1968. S. 125-141.

Daneš, František / Hlavsa, Zdeněk (1983): K vztahu aktuálního členění a sémantické stavby výpovědi. In: Slovo a slovesnost 44/1/1983. S. 3-11.

Dovalil, Vít / Káňa, Tomáš / Peloušková, Hana / Zbytovský, Štěpán / Vavř́n, Martin (2016): Korpus InterCorp - němčina, verze 9 z 9. 9. 2016. Praha: Ústav Českého národního korpusu FF UK. http://www.korpus.cz [10. 5. 2016].

Engel, Ulrich (1988): Deutsche Grammatik. Heidelberg, Julius Groos Verlag.

Eroms, Hans-Werner (1986): Funktionale Satzperspektive. Tübingen, Max Niemeyer Verlag.

Firbas, Jan (1982): „Aktuální členění větné“ (,) či „funkční perspektiva větná“? In: Slovo a slovesnost 43/4/1982. S. 282-293.

Firbas, Jan (1962): Ze srovnávacích studií slovosledných (K Mathesiovu pojetí slovosledné soustavy). In: Slovo a slovesnost 23/3/1962. S. 161-174.

Götze, Lutz (1989): Knaurs Grammatik der deutschen Sprache. Sprachsystem und Sprachgebrauch. München: Lexikographisches Institut.

Helbig, Gerhard / Buscha, Joachim (1990): Deutsche Grammatik. Ein Handbuch für den Ausländerunterricht. Berlin/München/Leipzig/Zürich/New York, Langenscheidt.

Hoberg, Ursula (1981): Die Wortstellung in der geschriebenen deutschen Gegenwartssprache. München, Max Hueber Verlag. 
Kunkel-Razum, Kathrin / Münzberg, Franziska (Hrsg.) (2005): DUDEN 4-Die Grammatik. Mannheim/Zürich, Dudenverlag.

Lötscher, Andreas (1983): Satzakzent und Funktionale Satzperspektive im Deutschen. Tübingen, Max Niemeyer Verlag.

Mathesius, Vilém (1947): Čeština a obecný jazykozpyt. Praha, Melantrich.

Peloušková, Hana (2016): Besetzung der Satzspitze im Deutschen und Tschechischen. In: Brünner Hefte zu Deutsch als Fremdsprache 9/2/2016. Brno: Masarykova univerzita. S. 101-117.

Peloušková, Hana (2009): Leitfaden zur Syntax des einfachen Satzes. Kurz gefasste Theorie mit Übungen und Ergänzungsmaterialien. Brno: Masarykova univerzita.

Sgall, Petr / Hajičová, Eva / Buráňová, Eva (1980): Aktuálni členěni věty v češtině. Praha, Academia.

Svoboda, Aleš (1989): Kapitoly z funkčni syntaxe. Praha, Státní pedagogické nakladatelství.

Štícha, František (2015): Německá srovnávaci gramatika. Praha, Academia.

Štícha, František / Vondráček, Miloslav / Kolářová, Ivana / Hoffmannová, Jana / Bílková, Jana / Svobodová, Ivana (2013): Akademická gramatika spisovné čestiny. Praha, Academia.

Štícha, František (Hrsg.) (2011): Kapitoly z české gramatiky. Praha, Academia.

Trávníček, František (1961): O tak zvaném aktuálním členění větném. In: Slovo a slovesnost 22/3/1961. S. 163-171.

Uhlírová, Ludmila (1987): Knižka o slovosledu. Praha, Academia.

Uhlírová, Ludmila (1983) Aktuální členění a styl jazykových projevů (na materiále z publicistických textů). In: Slovo a slovesnost 44/4/1983 S. 284-294.

Uhlírová, Ludmila (1969): Vztah syntaktické funkce větného členu a jeho místa ve větě (Metody a výsledky statistického zkoumání). In: Slovo a slovesnost 30/4/1969. S. 358-370.

Zifonun, Gisela / Hoffmann, Ludger / Strecker, Bruno (1997): Grammatik der deutschen Sprache. Band 2. Berlin / New York, Walter de Gruyter.

PhDr. Hana Peloušková, Ph.D.

Katedra německého jazyka a literatury

Pedagogická fakulta

Masarykova univerzita

Poříćí 9

60300 Brno

pelouskova@ped.muni.cz 